\title{
INFLUÊNCIA DA CONSCIÊNCIA FONOLÓGICA NA ESCRITA DE PRÉ-ESCOLARES
}

\section{Influence of phonological awareness on preschool children writing}

\author{
Adriane Bittencourt Dambrowski ${ }^{(1)}$, Cristine Leal Martins ${ }^{(2)}$, \\ Juliana de Lima Theodoro ${ }^{(3)}$, Erissandra Gomes ${ }^{(4)}$
}

\begin{abstract}
RESUMO
Objetivo: analisar a influência da consciência fonológica no estágio de desenvolvimento da escrita de crianças pré-escolares. Métodos: estudo clínico randomizado comparado. Aplicou-se o protocolo CONFIAS e um ditado de palavras, a uma amostra constituída de 57 crianças, divididas aleatoriamente em grupo intervenção (30) e grupo controle (27). A faixa etária foi de 5 anos e 1 mês a 6 anos e 6 meses. Para o $\mathrm{Gl}$ foram realizadas 10 intervenções de 30 minutos, com atividades lúdicas de consciência fonológica, enquanto que para o GC não foram realizadas atividades. Após, todas as crianças foram reavaliadas com os mesmos instrumentos. Resultados: na avaliação inicial, 92,6\% e 93,4\% das crianças do GC e GI respectivamente, eram pré-silábicas quanto ao nível de escrita, e apresentavam pontuações da consciência fonológica semelhantes, para o GC 26 e para o GI 24 acertos. Ao passo que na avaliação final, no Gl, $36,7 \%$ das crianças evoluíram para o nível silábico e 10\% evoluíram para o nível silábico-alfabético da escrita $(P<0,0001)$, contra apenas $14,8 \%$ e $3,7 \%$ respectivamente, das do $\mathrm{GC}(\mathrm{P}=0,056)$. Com relação ao nível de consciência fonológica, a evolução do $\mathrm{GI}$ ainda é mais marcante, as crianças passaram de 24 para 37 acertos na avaliação final $(P<0,0001)$, enquanto que os sujeitos do GC passaram de 26 para 28 pontos na avaliação final. Conclusão: a estimulação da consciência fonológica interferiu no estágio do desenvolvimento da escrita.
\end{abstract}

DESCRITORES: Estudos de Linguagem; Consciência; Aprendizagem

\section{INTRODUÇÃO}

As crianças aprendem as regras da linguagem ainda muito pequenas, apenas por meio do seu uso, sem instrução formal, pois o próprio meio é um fator significante para essa aprendizagem. Elas se apropriam do código e adquirem as particularidades da língua das pessoas que a cercam. Quando entram na pré-escola, já dominam sua língua nativa

(1) Fonoaudióloga Clínica do Instituto de Previdência e Assistência dos Servidores Municipais de Novo HamburgoRS; Especialista em Linguagem pelo CEFAC - Saúde e Educação.

(2) Fonoaudióloga;EspecializaçãoemLinguagempeloCEFACSaúde e Educação.

(3) Fonoaudióloga Clínica da Prefeitura dos Municípios de Machadinho e Maximiliano de Almeida-RS; Especialista em Linguagem pelo CEFAC - Saúde e Educação.

(4) Fonoaudióloga; Docente do Curso de Fonoaudiologia do Centro Universitário Metodista IPA; Mestre em Ciências Médicas: Pediatria pela Universidade Federal do Rio Grande do Sul. de forma competente, têm um vocabulário considerável e sabem usar as regras gramaticais ${ }^{1}$.

Por outro lado, a aquisição da linguagem escrita requer um ensino formal ${ }^{2}$. Ao comparar o fluxo da linguagem falada com o da escrita, percebe-se, que o primeiro é contínuo, enquanto que o fluxo da linguagem escrita é segmentado em unidades como sentenças, palavras e letras. A dificuldade das crianças é de estabelecer a relação entre os elementos contínuos da fala e os segmentos discretos da escrita ${ }^{3}$.

O sistema de escrita alfabético tem um número limitado de unidades (letras do alfabeto) que combinadas entre si de diferentes maneiras formam todos os vocábulos existentes de nossa língua. Essa característica da escrita alfabética permite a auto-aprendizagem pelo leitor, pois ao se deparar com uma palavra nova, ele a lerá por decodificação fonológica 4. O funcionamento desse sistema exige que os aprendizes estabeleçam as associações entre grafema e fonemas e isso envolve a capacidade de análise e síntese de fonemas da linguagem falada 5 . 
Durante os últimos anos, estudos nesta área têm demonstrado que para a criança aprender a ler é essencial que ela tenha habilidades de processamento fonológico ${ }^{6}$. A capacidade de refletir sobre a estrutura sonora da fala bem como manipular seus componentes estruturais, a chamada consciência fonológica, está intimamente relacionada à aprendizagem da leitura e escrita $2,4,7-12$. Assim, estudos comprovam que a introdução de atividades com rimas, aliterações, sílabas e fonemas na pré-escola possa produzir ganhos importantes no desenvolvimento de conceitos e habilidades que são prérequisitos no processo de alfabetização ${ }^{13}$.

Cabe ressaltar que este estudo fundamenta-se na teoria psicogenética ${ }^{14}$ que diz respeito ao desenvolvimento da escrita, descreve os estágios pelos quais as crianças passam até compreender o ler e o escrever. A teoria centra-se no ato de aprender por meio da construção de um conhecimento que é realizado pelo educando. Ele é visto como um agente e não como um ser passivo que recebe e absorve o que lhe é ensinado. Os conceitos de prontidão, maturidade, habilidades motoras e perceptuais não são vistos isoladamente, mas vinculados ao contexto da realidade sócio-cultural do aluno. Eles podem explicar as diferenças individuais e os diferentes ritmos dos alunos.

Baseando-se no que foi exposto, este estudo tem por objetivo pesquisar a influência da consciência fonológica no nível de desenvolvimento da escrita de crianças pré-escolares.

\section{MÉTODOS}

O estudo teve caráter dinâmico, prospectivo, experimental, analítico, delineando-se como ensaio clínico randomizado comparado. A pesquisa foi realizada em duas escolas da rede municipal de ensino do Rio Grande do Sul (RS), sendo uma na cidade de Machadinho e outra na cidade de Novo Hamburgo, no período de agosto a dezembro de 2006.

Envolveram-se nesta pesquisa 57 crianças, sendo que 30 participaram do Grupo Intervenção (GI) e 27 do Grupo Controle (GC). Na cidade de Machadinho, 11 crianças faziam parte do GC e 12 do GI, sendo totalizadas 23 crianças nesta cidade. Em Novo Hamburgo, 16 crianças participaram do $\mathrm{GC}$ e 18 do $\mathrm{Gl}$, totalizando 34 crianças. A idade das crianças integrantes da pesquisa, variou de 5 anos e 1 mês a 6 anos e 6 meses.

O processo de amostragem teve como objetivo selecionar os sujeitos pré-escolares, seguindo os seguintes critérios de inclusão: crianças sem queixas de distúrbios visuais, auditivos, de linguagem e aprendizagem, de ambos os sexos, não-alfabetizados, indicados pelas professoras titulares, sem uma avaliação formal. Após a seleção das crianças, foi realizado um sorteio aleatório para definir quais crianças participariam do grupo controle (GC) e quais fariam parte do grupo intervenção (Gl).

Para a análise dos estágios de escrita neste estudo, foi utilizada a classificação proposta na literatura ${ }^{14}$, a qual refere os níveis de escrita como pré-silábico (não há relação entre a oralidade e a escrita), silábico (inicia-se uma relação entre a palavra falada e o uso de letras para representar seus segmentos), silábico-alfabético (ao escrever uma palavra o uso das letras corresponde de forma mais precisa aos segmentos desta palavra) e alfabético (a utilização das letras, ao escrever uma palavra, respeita o padrão ortográfico).

Todas as crianças foram avaliadas individualmente quanto ao grau de consciência fonológica, através da utilização do Protocolo CONFIAS ${ }^{13}$, para o qual, a pontuação máxima esperada é de 70 pontos no geral, sendo que para o nível de sílaba são esperados 40 pontos e para o fonema 30 pontos. Para a avaliação de escrita, foi realizado um ditado de palavras, não baseado em protocolo padronizado, elaborado pelas pesquisadoras, no qual era fornecido à criança duas folhas, uma contendo figuras com partes do corpo (pé, mão, dedo, nariz, cabelo, orelha, sobrancelha) e outra de animais (cão, sapo, urso, mosca, girafa, cavalo, elefante). O ditado era realizado utilizando-se apoio visual (figuras) e auditivo (as palavras eram faladas oralmente pelas pesquisadoras, uma a uma, ditando-as de modo como são pronunciadas normalmente nas conversações espontâneas, não forçando a pronúncia artificial das palavras, e sem o uso de gravação). A criança escrevia a palavra no espaço destinado, ao lado da figura, sem que fosse realizado nenhum tipo de interferência na avaliação.

As 30 crianças do $\mathrm{Gl}$ foram estimuladas com atividades lúdicas dirigidas de consciência fonológica, em grupo, em 10 momentos de 30 minutos cada, uma vez por semana. Após as 10 intervenções, todas as crianças (GI e GC) foram reavaliadas individualmente, quanto ao grau de consciência fonológica e ao nível de escrita, seguindo a mesma metodologia da avaliação inicial.

As atividades foram organizadas abrangendo os diversos segmentos da consciência fonológica, oportunizando a estimulação da consciência fonológica para o desenvolvimento do nível de escrita, baseando-se em atividades de outros autores ${ }^{15-17}$.

No primeiro e segundo encontro foram realizadas atividades sobre consciência silábica. As tarefas incluíram segmentar, contar, comparar, adicionar, subtrair, e transpor sílabas em palavras. $\mathrm{Na}$ terceira e quarta intervenção, foram aplicadas atividades que visavam trabalhar rimas e aliteração 
(identificação e produção). No quinto e sexto encontro, trabalhou-se a noção de palavra. As tarefas compreenderam a identificação e a substituição de palavra em sentenças. Na sétima e oitava semana, houve atividades voltadas ao treino da consciência fonêmica. Da mesma forma que nas primeiras intervenções, foram feitas atividades identificando os fonemas, contando, subtraindo, adicionando e transpondo-os nas palavras. Nas duas últimas intervenções enfatizou-se o treinamento de correspondências grafema-fonema, para cada letra do alfabeto e a revisão das atividades propostas durante todo o trabalho, respectivamente.

A análise estatística foi executada no software Statistical Package for Social Science (SPSS) 10.0 for Windows. A análise descritiva das variáveis quantitativas foi realizada por meio da observação do cálculo de médias e desvio padrão, bem como de mediana acompanhada de seu intervalo interquartil. Para as demais variáveis, qualitativas, foram calculadas a freqüência absoluta. Todos os testes foram realizados na forma bi-caudal, admitindo-se como estatisticamente significativos os valores de $P<0,05$. O teste de Friedman foi utilizado para comparar as variáveis de nível de escrita. As variáveis de consciência fonológica foram analisadas de forma emparelhada, através do teste de Wilcoxon. O teste $t$ foi utilizado comparar a idade entre os grupos controle e intervenção.

Esta pesquisa foi aprovada pelo Comitê de Ética em Pesquisa do CEFAC - Saúde e Educação, sob o número 132/06 e o considerou aprovado sem risco, com necessidade do Termo de Consentimento Livre e Esclarecido. Os pesquisadores assinaram um Termo de Compromisso de Utilização e Divulgação de Dados, os pais e/ou responsáveis das crianças assinaram um Termo de Consentimento Livre e Esclarecido e as escolas envolvidas assinaram o Termo de Autorização Institucional.

\section{RESULTADOS}

A idade média das crianças do GC foi maior do que as crianças do GI $(69,70$ meses para o CG e 69,43 meses para o Gl), entretanto sem significância estatística (Tabela 1).
Os níveis de escrita ${ }^{14}$ encontrados na avaliação inicial e final de todas crianças da pesquisa, assim como dos GC e Gl, encontram-se detalhados na Tabela 2. Em ambos grupos (GC e GI), nenhuma das crianças alcançou o nível alfabético de escrita. No $G C$ o valor de $P$ foi limítrofe $(P=0,056)$ e no $G l$ estatisticamente significativo $(P<0,0001)$.

Os dados encontrados quanto à pontuação da consciência fonológica são estatisticamente significativos, 25 pontos na avaliação inicial e 33 na avaliação final, no que se refere ao somatório total do teste, quando comparados todos os sujeitos na avaliação inicial e final. Já quando comparados os $\mathrm{GC}$ e Gl, pode-se perceber que o $\mathrm{Gl}$ apresentou maior pontuação, 37 pontos (dados estatisticamente significativos) na avaliação final, ou seja, comparando com o GC, que não apresentou uma evolução significativa no nível de consciência fonológica (Tabela 3).

Todas as crianças envolvidas na pesquisa progrediram nas habilidades fonológicas, entretanto, comparando-se o Gl que sofreu intervenção com o GC, verifica-se que a evolução do primeiro foi superior.

A maioria das crianças do Gl apresentou evolução significativa, tanto na escrita (na avaliação final $53,3 \%$ estavam no nível pré-silábico, $36,7 \%$ no silábico e 10\% silábico-alfabético), quanto na consciência fonológica (de 24 para 37 pontos) proporcionando o conhecimento destes conceitos e habilidades de consciência fonológica para algumas crianças e um desafio para as mais avançadas, de aprimorarem suas habilidades, com posterior evolução na escrita.

As crianças do GC não participaram da estimulação da consciência fonológica, portanto obtiveram, nas avaliações finais, resultados similares a das avaliações iniciais para o mesmo grupo.

\section{DISCUSSÃO}

Como se pode observar nas Tabelas 2 e 3, há uma diferença significativa entre as avaliações das crianças do GC e Gl. Na avaliação inicial da escrita e da consciência fonológica, as crianças apresentavam níveis de escrita e pontuações da consciência fonológica semelhantes em ambos grupos,

Tabela 1 - Variáveis quantitativas comparadas entre GC e GI

\begin{tabular}{lccc}
\hline Variável & $\begin{array}{c}\text { GC } \\
\mathrm{n}=27\end{array}$ & $\begin{array}{c}\text { GI } \\
\mathrm{n}=30\end{array}$ & Valor $\mathbf{P}$ \\
\hline Idade (em meses) & $69,70 \pm 4,47$ & $69,43 \pm 5,06$ & 0,832 \\
\hline $\mathrm{n}=57$, média \pm desvio padrão, teste $\mathrm{t}, \mathrm{p}<0,05$ & & &
\end{tabular}


Tabela 2 - Comparação do nível de escrita do GC e GI: avaliação inicial e avaliação final

\begin{tabular}{lccc}
\hline Grupo & Avaliação Inicial & Avaliação Final & Valor P \\
\hline GC & $25(92,6 \%)$ & $22(81,5 \%)$ & \\
$\quad$ Pré-Silábico & $2(7,4 \%)$ & $4(14,8 \%)$ & $\mathrm{P}=0,056$ \\
Silábico & $0(0,0 \%)$ & $1(3,7 \%)$ & \\
Silábico-Alfabético & & & \\
\hline GI & $28(93,4 \%)$ & $16(53,3 \%)$ & $\mathrm{P}<0,0001$ \\
Pré-Silábico & $1(3,3 \%)$ & $11(36,7 \%)$ & \\
Silábico & $1(3,3 \%)$ & $3(10 \%)$ & \\
Silábico-Alfabético & & & \\
\hline GC e GI & $53(93,0 \%)$ & $38(66,7 \%)$ & $\mathrm{P}<0,0001$ \\
$\quad$ Pré-Silábico & $3(5,3 \%)$ & $15(26,3 \%)$ & \\
Silábico & $1(1,8 \%)$ & $4(7,0 \%)$ & \\
Silábico-Alfabético & & & \\
\hline
\end{tabular}

Teste de Friedman, $\mathrm{p}<0,05, \mathrm{GC}=$ grupo controle, $\mathrm{Gl}=$ grupo intervenção

Tabela 3 - Comparação da consciência fonológica do GC e Gl: avaliação inicial e avaliação final

\begin{tabular}{|c|c|c|c|}
\hline & Avaliação Inicial & Avaliação Final & Valor $\mathbf{P}$ \\
\hline \multicolumn{4}{|l|}{ GC } \\
\hline Nível de sílaba & 21 (14 a 28) & 22 (13 a 27) & 0,987 \\
\hline Nível de fonema & $5(2$ a 10$)$ & 7 (3 a 8) & 0,298 \\
\hline Soma total & 26 (19 a 35) & $28(18$ a 35$)$ & 0,593 \\
\hline \multicolumn{4}{|l|}{ GI } \\
\hline Nível de sílaba & 21 (16 a 28) & 28 (20 a 30) & 0,002 \\
\hline Nível de fonema & 3,5 (1 a 9) & 10 (6 a 12) & $<0,0001$ \\
\hline Soma total & 24 (18 a 36) & 37 (26 a 42) & $<0,0001$ \\
\hline \multicolumn{4}{|l|}{ GC e GI } \\
\hline Nível de sílaba & $21(15,5$ a 28$)$ & $25(18,5$ a 28$)$ & 0,009 \\
\hline Nível de fonema & $4(1,5$ a 9) & $8(5$ a 11) & $<0,0001$ \\
\hline Soma total & $25(18$ a 35,5$)$ & $33(24$ a 40$)$ & $<0,0001$ \\
\hline
\end{tabular}

Mediana (intervalo interquartil), teste de Wilcoxon, $\mathrm{p}<0,05, \mathrm{GC}=$ grupo controle, $\mathrm{Gl}=$ grupo intervenção

ou seja, 25 crianças do GC (92,6\%) e 28 crianças do GI $(93,4 \%)$ eram pré-silábicas, e alcançaram na consciência fonológica, 26 pontos e 24 pontos respectivamente. Já na avaliação final o resultado foi diferente, pois o GC não apresentou evolução significativa na escrita, mantendo-se a maior parte das crianças no nível pré-silábico $(81,5 \%)$, assim como manteve pontuação similar a avaliação inicial na consciência fonológica (aumento de 2 pontos na avaliação final). As evoluções apresentadas no GC são atribuídas à aprendizagem escolar, maturação ou possível efeito cumulativo da retestagem ${ }^{4}$.

A melhora nos resultados do GI (evolução no nível de escrita e no desempenho no Teste CONFIAS), deve-se ao programa de estimulação da consciência fonológica, pois as crianças do Gl apresentaram uma evolução significativa tanto ao nível de escrita quanto de consciência fonoló- gica, o que não aconteceu no GC. Assim como em nesse trabalho, estudos experimentais mostraram que as crianças que receberam treinamento de consciência fonológica, apresentaram habilidades para leitura significativamente mais altas do que as crianças que não sofreram esta estimulação, comprovando que as habilidades da consciência fonológica influenciam na leitura, havendo uma associação entre a consciência fonológica e a aquisição do letramento $7,18,19$.

Os achados desta pesquisa corroboram com achados de outro estudo, realizado também com pré-escolares, no qual se verificou a mesma relação entre consciência fonológica e escrita, entretanto realizado com crianças de idades diferentes ${ }^{8}$. As crianças dos $\mathrm{GC}$ e $\mathrm{Gl}$ apresentavam médias diferentes de idade, e mesmo com esta diferença, os resultados foram significativos para o 
GI, ficando limítrofe para o GC em relação ao nível de escrita.

Em outro estudo ${ }^{19}$, mais recente, que teve objetivo semelhante ao da presente pesquisa, realizado com 59 crianças, utilizando o mesmo teste, verificou-se que o grupo pesquisa apresentou mediana de acertos superior ao grupo controle, no nível de consciência fonológica na reavaliação. Os dados da pesquisa corroboram com os deste estudo, em que o Gl obteve mediana 37,00 comparado ao GC, em que a mediana foi de 28,00 , também houve diferença significativa entre o Gl e o GC no nível de escrita $(P<0,0001)$.

Uma pesquisa realizada ${ }^{12} \mathrm{com}$ uma população de 33 pré-escolares, avaliados quanto ao nível de escrita e consciência fonológica, apresentou achados semelhantes em relação à escrita e desempenho na consciência fonológica. Tal achado encontra-se em sintonia com os da presente pesquisa, onde a correlação do nível de escrita e consciência fonológica também foi observada. As crianças pré-silábicas demonstraram grau restrito de consciência fonológica, crianças silábicas e as silábicoalfabéticas demonstraram um maior aprimoramento da fase da escrita e desempenho na consciência fonológica, demonstrando a inter-relação da consciência fonológica e a aquisição do código escrito, comprovando que a consciência fonológica é uma habilidade de suma importância na aquisição do letramento.

Um estudo realizado ${ }^{7}$ evidenciou que a terapia em consciência fonológica, associada ao ensino da correspondência fonema-grafema, interferiu no processo de alfabetização, facilitando a aquisição do código alfabético. Nesse estudo estas atividades também oportunizaram um melhor desempenho na consciência fonológica e evolução no nível de escrita observado na avaliação inicial, principalmente no Gl que foi estimulado, porém sem a intenção de alfabetizar as crianças pré-escolares.

Em um estudo longitudinal, com o propósito de verificar se crianças pré-escolares, sem nenhum conhecimento de leitura e soletração, apresentavam consciência fonológica ao ingressarem no ensino formal e se a presença dessa habilidade favorecia a aquisição da leitura e escrita. Os achados finais comprovaram que existe uma relação entre a consciência fonológica e desempenho em leitura e escrita, que a intervenção na educação infantil seria o momento adequado para a aquisição e o desenvolvimento das habilidades metafonológicas e que, o desempenho em consciência fonológica seria um bom indicador de sinais de risco para distúrbios de leitura e escrita ${ }^{20}$. Os dados encontrados neste e em outros estudos ${ }^{21-23}$, assim como os da presente pesquisa novamente mostram que, existe relação causal entre a consciência fonológica e desenvolvimento da escrita, e que estas habilidades deveriam de ser abordadas na educação infantil, ou seja, no início da escolarização, possibilitando um maior domínio dos fonemas e associação posterior destes aos grafemas, auxiliando na aquisição da lecto-escrita e diminuindo os índices de insucesso escolar.

Esses dados sugerem que um programa padronizado de estimulação da consciência fonológica para pré-escolares auxilia na sua prontidão para a alfabetização ${ }^{4,7-9,11,24-26}$. Após as análises dos resultados obtidos nesta pesquisa, e comparando-se com os estudos acima, entre outros 5,6,8,9,12,24,25,27-29, observou-se que existe influência da consciência fonológica no nível de escrita de crianças pré-escolares.

Verifica-se em outros estudos ${ }^{17,24,27,29}$ que nas crianças que têm dificuldade de leitura e escrita, os níveis de consciência fonológica encontram-se precários. Assim, pode-se inferir que as causas do fracasso escolar podem não ser somente os fatores neuroanatomofisiológicos e sócio-econômico-culturais, mas também as habilidades metafonológicas. A realidade de ensino brasileira deveria privilegiar instruções metafonológicas preparatórias, para atingir maior grau de competência da leitura e escrita, pois, sabe-se que alfabetizar melhor e no tempo certo é o método mais eficaz e mais econômico ${ }^{17,30}$.

Por outro lado, mesmo em crianças com dificuldades de leitura já instaladas, estudos têm demonstrado que o treino explícito da consciência fonológica pode melhorar a performance na leitura. Em um estudo realizado em Hong Kong ${ }^{31}$, observou-se que 5 dias de treinamento da consciência fonológica bastaram para melhorar a leitura das crianças. Pesquisas ${ }^{18,21,22}$ comprovam que as habilidades de consciência fonológica são importantes para reduzir o risco de alterações na leitura, tanto em crianças com risco para a dislexia quanto em crianças com alterações na fala.

Com base nestes dados, sugere-se que a atuação do fonoaudiólogo como consultor escolar, elaborando programas para o desenvolvimento da consciência fonológica é de grande valia para as escolas, proporcionando aos alunos, através de atividades lúdicas a aquisição de habilidades fundamentais para a leitura e escrita. Sugere-se a continuidade de estudos nesta área, utilizando-se de amostras ampliadas para um maior aprofundamento das questões levantadas. 


\section{CONCLUSÃO}

Após a análise dos dados obtidos neste estudo, verificou-se que há influência da consciência fonológica no nível de escrita de crianças pré-escolares, e, que a estimulação da consciência fonológica em pré-escolares auxilia na evolução do nível de escrita.

O estudo teve a finalidade de demonstrar que a consciência fonológica é vital para a aquisição do código escrito, otimizando o processo de alfabetização, assim como, demonstrar que as crianças que são estimuladas com atividades de consciência fonológica apresentam melhor desenvolvimento na escrita.

\section{AGRADECIMENTOS}

Nossos sinceros agradecimentos as Secretarias de Educação das cidades de Machadinho e Novo Hamburgo, permitindo que esta pesquisa fosse realizada.

A disponibilidade e incentivo da equipe diretiva das Escolas Municipais envolvidas na pesquisa.

Aos pais e responsáveis das crianças participantes da pesquisa, sem as quais nada disto seria possível.

Às crianças, que nos ensinaram como o papel do educador é essencial para o crescimento deste país, e o quanto nós fonoaudiólogos podemos participar deste processo educacional.

\section{ABSTRACT}

Purpose: to analyze the influence of the phonological awareness on preschool children writing. Methods: a randomized comparison study in which 57 children were evaluated in two aspects, namely: the level of phonological awareness (through CONFIAS protocol of phonological awareness) and the level of writing (through word dictation). They were split, randomly, in two different groups: the intervention group (30 children) and the control group ( 27 children). The age group was from 5 years and 1 month to 6 years and 6 months old. In the intervention group a schedule of 10 play sessions was set up based on phonological awareness activities. Meanwhile, the other group did not have any special activities. Thereafter, all subjects were again assessed with the same tests. Results: in the initial evaluation, $92.6 \%$ and $93.4 \%$ of the children in the control group and intervention group, respectively, were in the pre-syllabic writing level. Both groups had similar scores in the phonological awareness test, 26 points in the control group and 24 in the intervention group. Although, in the final evaluation $36.7 \%$ of the subjects on the intervention group achieved the syllabic level of writing and $10 \%$ reached the syllabic-alphabetical level of writing $(\mathrm{P}<0.0001)$; against, just $14.8 \%$ and $3.7 \%$, respectively, on the control group $(\mathrm{P}=0.056)$. As long as the phonological awareness is concerned the children on the intervention group had an even more dramatic progress, they went from 24 points in the first evaluation to 37 points in the final $(P<0.0001)$. Meanwhile, the subjects in the control group went from 26 to 28 points in the final evaluation. Conclusion: the phonological awareness practice interfered in the writing level development.

KEYWORDS: Language Arts; Conscience; Learning

\section{REFERÊNCIAS}

1. França MP, Wolff CL, Moojen S, Rotta NT. Aquisição da linguagem oral: relação e risco para a linguagem escrita. Arq Neuropsiquiatr. 2004; 62(2b):469-72.

2. Navas ALGP, Santos MTM. Aquisição e desenvolvimento da linguagem escrita. In: Navas ALGP, Santos MTM. Distúrbios de leitura e escrita: teoria e prática. São Paulo: Manole; 2002.

3. Byrne B. Treinamento da consciência fonêmica em crianças pré-escolares: por que fazê-lo e qual seu efeito? In: Cardoso-Martins C, organizador.
Consciência fonológica e alfabetização. Petrópolis: Vozes; 1996.

4. Capovilla AGS, Capovilla F. Efeito do treino de consciência fonológica em crianças com baixo nível sócio-econômico. Psicol Reflex Crit. 2000; 13(1):7-24.

5. BarreraSD, MalufMR. Consciênciametalingüística e alfabetização: estudo com crianças da primeira série do ensino fundamental. Psicol Reflex Crit. 2003; 16(3):491-502.

6. Capellini SA, Ciasca SM. Aplicação da prova de consciência fonológica (PCF) em escolares com dificuldades na leitura. J Bras Fonoaudiol. 1999; 1(1):11-4. 
7. Paula GR, Mota HB, Keske-Soares M. A terapia em consciência fonológica no processo de alfabetização. Pró-Fono. 2005; 17(2):175-84.

8. Barrera SD, Maluf MR. Consciência fonológica e linguagem escrita em pré-escolares. Psicol Reflex Crit. 1997; 10(1):125-45.

9. Cárnio MS, Santos D. Evolução da consciência fonológica em alunos de ensino fundamental. PróFono. 2005; 17(2):195-200.

10. Papis L, Assencio-Ferreira VJ. Consciência fonológica como meio de avaliação de crianças com dificuldades de aprendizagem escolar. Rev CEFAC. 2001; 3(2):117-21.

11. Paes CTS, Pessoa ACRG. Habilidades fonológicas em crianças não alfabetizadas e alfabetizadas. Rev CEFAC. 2005; 7(2):149-57.

12. Santamaria VL, Leitão PB, Assencio-Ferreira VJ. A consciência fonológica no processo de alfabetização. Rev CEFAC. 2004; 6(3):237-41.

13. Moojen $S$, organizador. Consciência fonológica Instrumento de avaliação seqüencial. São Paulo: Casa do Psicólogo; 2003.

14. Ferreiro $E$, Teberosky A. Psicogênese da língua escrita. 4. ed. Porto Alegre: Artes Médicas; 1991.

15. Adams MJ, Foorman BR, Lundberg I, Beeler T. Consciência fonológica em crianças pequenas. Porto Alegre: Artmed; 2006.

16. Almeida EC, Duarte PM. Consciência fonológica: atividades práticas. Rio de Janeiro: Revinter; 2003. $102 p$.

17. Capovilla AGS, Capovilla F. Alfabetização: método fônico. 3. ed. São Paulo: Memnon; 2004.

18. Blaiklock KE. The importance of letter knowledge in the relationship between phonological awareness and reading. J Res Read. 2004 feb; 27(1):36-57.

19. Britto DBO, Castro CD, Gouvêa FG, Silveira OS. A importância da consciência fonológica no processo de aquisição e desenvolvimento da escrita. Rev Soc Bras Fonoaud. 2006; 11(3):142-50.

20. Pestun MSV. Consciência fonológica no início da escolarização e o desempenho ulterior em leitura e escrita: estudo correlacional. Est Psicol. 2005; 10(3):27-37.

21. Carroll JM, Snowling MJ. Language and phonological skills in children at high risk of reading difficulties. J Child Psychol Psych. 2004; 45(3):631-40.

22. Segers E, Verhoeven L. Long-term effects of computer training of phonological awareness in kindergarten. J Computer Assisted Learning. 2005; 21(1):17-27.

23. Share DL. Phonological recoding and selfteaching: sine qua non of reading acquisition. Cognition.1995; 55(2):151-218.

24. Guimarães SRK. Dificuldades no desenvolvimento da lectoescrita: o papel das habilidades metalingüísticas. Psicol Teor Pesq. 2002; 18(3):247-59.

25. Valdivieso LB. La Conciencia fonologica como uma zona de desarollo proximo para el aprendizaje inicial de la lectura. Estúdios Pedagógicos. 2002; 28:165-77.

26. Passenger T, Stuart M, Terrell C. Phonological processing and early literacy. J Res Read. 2000; 23(1):55-66.

27. Dourado MB, Schmid E, Chiappetta ALML. Consciência fonológica em disléxicos. Rev CEFAC. 2005; 7(4):415-8.

28. Read C, Zhang Y, Nie H, Ding B. The ability to manipulate speech sounds depends on knowing alphabetic writing. Cognition. 1986; 24:31-44.

29. Demont E. Developmental dyslexia and sensitivity to rhymes: a perspective remediation. Curr Psychol Letters. 2003; 10(1):212-32.

30. Sprugevica I, Hoien T. Early phonological skills as a predictor of reading acquisition: a follow-up study from kindergarten to the middle of grade 2 . Scand J Psychol. 2003; 44(2):119-24.

31. Suk-Han Ho C, Nga-Lun Ma R. Training in phonological strategies improves Chinese dyslexic children's character reading skills. J Res Read. 1999; 22(2):131-42.

\section{RECEBIDO EM: 09/07/2007}

ACEITO EM: 22/04/2008

Endereço para correspondência:

Av. Pedro Adams Filho, 4790/205

Novo Hamburgo - RS

CEP: 93320-006

Tel: (51) 3593-1991

E-mail: abdfono@uol.com.br 